POS $\quad \begin{aligned} & \text { PROCEEDINGS } \\ & \text { OF SCIENCE }\end{aligned}$

\title{
Recent CMS results on the Soft QCD \& Forward Physics
}

\author{
Ankita Mehta \\ (On behalf of the CMS Collaboration) \\ MTA-ELTE Lendület CMS Particle and Nuclear Physics Group, Eötvös Loránd University, \\ Budapest, Hungary \\ E-mail: ankita.mehta@cern.ch
}

This presentation covers the recent measurements on Soft QCD and Forward Physics by CMS experiment. 


\section{Introduction}

An overview of recent results from forward and small-x QCD physics program at the CMS experiment [1] is provided. These studies are based on the events produced by strong interactions of partons inside the hadrons, which involve low momentum transfer, for which predictions of quantum chromodynamics cannot be obtained perturbatively. Measurements are sensitive to the transition region between hard processes calculable with perturbative techniques and soft processes described by nonperturbative models. Thus, these measurements are required for a full description of particle production in proton-proton (pp) collisions at the LHC provide and also provide an important input for the tuning of Monte Carlo (MC) event generators.

\section{Measurement of the average very forward energy as a function of the track multiplicity at central rapidities in proton-proton collisions at $\sqrt{s}=13 \mathrm{TeV}$}

The energy carried by particles produced in the very forward region $(-6.6<\eta<-5.2)$ covered by the CASTOR calorimeter [2] of the CMS experiment is a powerful probe of the activity of the underlying event [3]. The measurements presented here provide the first correlation study of hadron activity at very forward and central rapidities performed at $\sqrt{s}=13 \mathrm{TeV}$. The reported analysis is performed based on $0.22 \mathrm{nb}^{-1}$ of low-luminosity data recorded using the CMS detector at $0 \mathrm{~T}$ magnetic field. The events are categorized according to the number of reconstructed tracks. The average total, electromagnetic, and hadronic energy per event is calculated for each track multiplicity $\left(\mathrm{N}_{\mathrm{ch}}\right)$ bin. The total energy deposited in CASTOR is obtained by summing the energy measured in each calorimeter tower above the noise threshold.

The data are compared to a broad range of model predictions covering different parameter tunes as well as entirely different physics approaches. The models considered are PYTHIA8 [4] with tunes CUETP8M1 [5], and 4C [6], combined with the MBR [7] model to describe diffractive processes. The data are also compared to the predictions of EPOS LHC [8] and SIBYLL 2.1 [9]. Furthermore, predictions by QGSJETII.04 [10], SIBYLL 2.3c [11], PYTHIA8 tune CP5 [12], and HERWIG 7.1 [13,14] with the default tune for soft interactions [15] are also compared to the data.

Figure 1 shows the average total energy measured in CASTOR, which increases with $\mathrm{N}_{\mathrm{ch}}$. This feature is consistent with the general behaviour of the underlying event measured at central rapidities [16] and is reproduced by all models considered. All models describe these data with minor tensions only. Thus, the model parameter tunes for the underlying event, as determined at central rapidities, are consistent with the very forward data within experimental uncertainties.

The measured ratio of the average electromagnetic and hadronic energies is shown in the Fig. 2, which is almost constant over the whole multiplicity range. This measured ratio depends on the details of hadronization in the observed phase space. Deviations of model predictions from the data hint on underlying differences of final state hadron production mechanisms contributing to the observed average energies. The observed independence of the measured ratio from $\mathrm{N}_{\mathrm{ch}}$ indicates that no dramatic change of the particle production mechanism is observed at this very forward pseudorapidity. All model predictions are lower than the data, specifically those of the modern tunes, PYTHIA8 tune CP5 and SIBYLL 2.3c, whereas the QGSJETII.04, SIBYLL 2.1, and HERWIG 7.1 models give the best description of the ratio within the systematic uncertainties. 

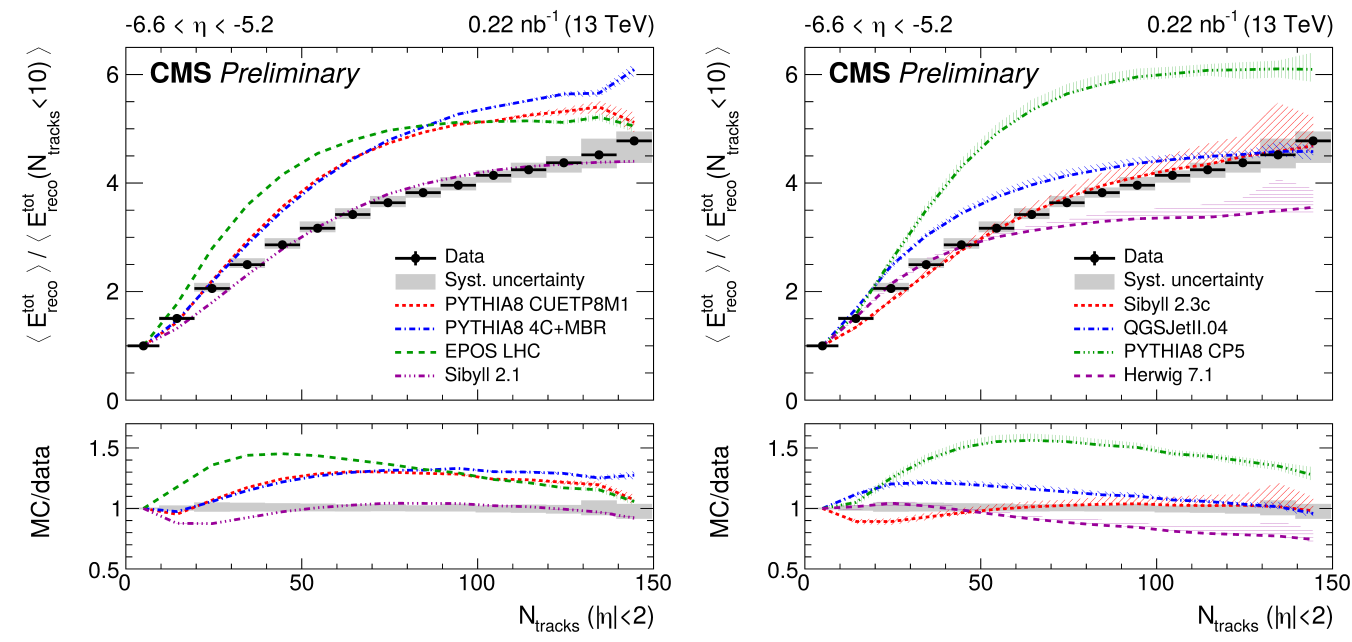

Figure 1: Average total energy reconstructed in the CASTOR calorimeter normalised to that in the first bin $\left(\mathrm{N}_{\mathrm{ch}}<10\right)$ as a function of the number of reconstructed tracks for $|\eta|<2$. The data are shown as black circles and the corresponding systematic uncertainties with a gray band. The predictions of various event generators are compared to the data and the bands associated with the model predictions illustrate the model uncertainty [3].
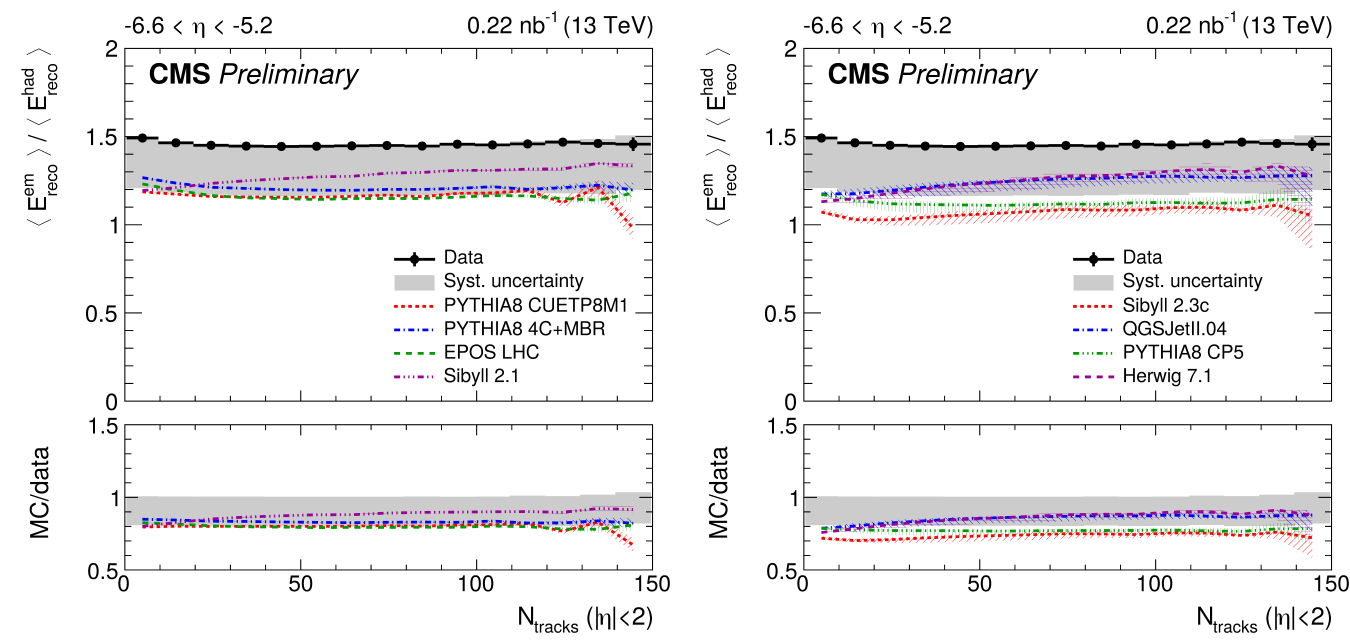

Figure 2: Ratio of average electromagnetic and hadronic energies reconstructed in the CASTOR calorimeter as a function $\mathrm{N}_{\mathrm{ch}}$ for $|\eta|<2$ [3].

\section{Evidence for $\mathrm{WW}$ production from double-parton interactions in proton-proton collisions at $\sqrt{s}=13 \mathrm{TeV}$}

The DPS cross section is measured for the first time using the same-sign $\mathrm{W}$ boson pair production with two $\mathrm{W}$ bosons decaying leptonically into a dimuon or an electron-muon pair [17]. The analyzed data corresponds to an integrated luminosity of $77.4 \mathrm{fb}^{-1}$ collected during 2016 and 2017. The production of same-sign WW via DPS has been studied in the past by the CMS Collaboration using pp collisions data at $\sqrt{s}=8 \mathrm{TeV}$, and an upper limit of $0.32 \mathrm{pb}$ was set on its production 
cross section at $95 \%$ confidence level [18]. The dominant background contributions stem from WZ production and jets misidentified as leptons. A multivariate analysis based on boosted decision trees (BDT) is used to discriminate the signal and background processes. Two different BDTs are trained using a set of lepton kinematic variables, defined based on the topological differences between the DPS signal and background processes. The two BDT distributions are mapped into a single variable which is then used to extract the DPS signal strength. To maximize the sensitivity of the signal process, the maximum likelihood fit $[19,20,21]$ is performed by categorizing the shape of final discriminant into different flavor and charge configurations of the final state leptons.
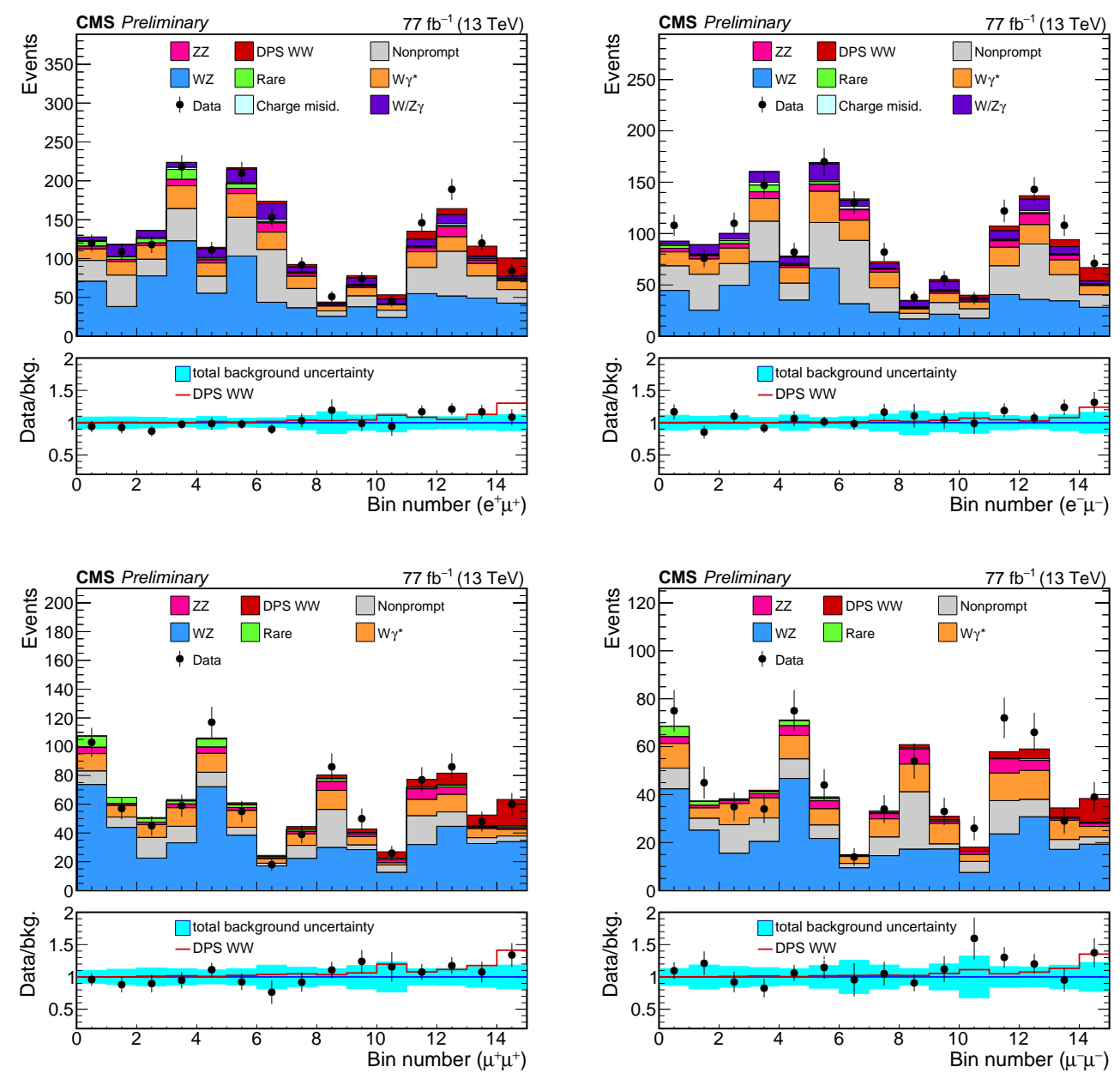

Figure 3: Distribution of the final BDT classifier output for $\mathrm{e} \mu$ (top) and $\mu \mu$ (bottom) final states, in the positive (left) and negative (right) charge configurations. Observed data are shown in black markers while the backgrounds and signal are shown in colored histograms with their postfit yields. The SHS WW and WWW contributions are grouped as the "Rare" background. The bottom panels show the ratio of data to the sum of all background contributions in the black markers along with the signal shown using a red line. The shaded band on the ratio plot represents the postfit background uncertainty, which includes both the statistical and systematic components [17].

Figure 3 shows the distributions of the final discriminant after performing a maximum likelihood fit to four different lepton flavor and charge configurations. The obtained results are compared 
with the predictions from PYTHIA8 and those obtained using the factorization approach [22] with a $\sigma_{\text {eff }}$ of $20.7 \pm 6.6 \mathrm{mb}$ as measured in $\mathrm{W}+2$ jets final state at $7 \mathrm{TeV}$ [23].
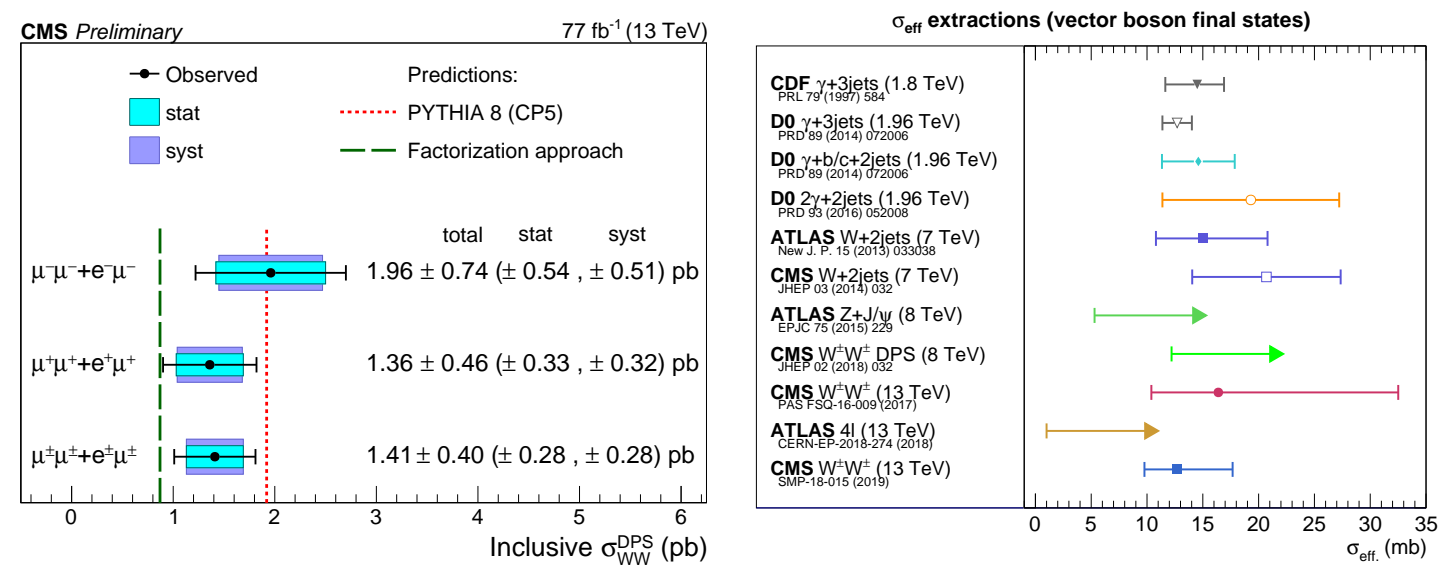

Figure 4: Observed cross section values for inclusive DPS WW production from the two lepton charge configurations and their combination (left). These values are obtained from the extrapolation of the observed DPS $\mathrm{W}^{ \pm} \mathrm{W}^{ \pm}$cross section to the inclusive $\mathrm{WW}$ case. The statistical and systematical uncertainties are shown using shaded bands. The predictions from PYTHIA8 and the factorization approach are represented using red-dotted and green-dashed lines, respectively. A summary of $\sigma_{\text {eff }}$ measurements from different experiments at different collision energies (right). The data points are taken from the references quoted in the figure [17].

Figure 4 shows the obtained value of WW production cross section via DPS using ++ and final states along with their combination (left). Also shown is the value of $\sigma_{\text {eff }}$ and a comparison with previous measurements at different center-of-mass energies (right). All the measurements are compatible but within large systematic uncertainties warranting further detailed studies using more data and improved analysis techniques.

\section{Acknowledgments}

The author wishes to thank for their support the Hungarian Academy of Sciences "Lendület" (Momentum) Program (LP 2015-7/2015) and the National Research, Development and Innovation Office of Hungary (K 124845 and K 128713).

\section{References}

[1] CMS Collaboration, JINST 3 S08004 (2008).

[2] V. Andreev et al., Eur. Phys. J. C 67601 (2010).

[3] CMS Collaboration, CMS-PAS-FSQ-18-001 (2019), CDS Record 2669256.

[4] T. Sjöstrand et.al., Comput. Phys. Commun. 178, 852 (2008).

[5] CMS Collaboration, Eur. Phys. J. C 76, 155 (2016).

[6] R. Corke and T. Sjostrand, JHEP 1103, 032 (2011). 
[7] R. Ciesielski and K. Goulianos, PoS ICHEP 2012301 (2013).

[8] T. Pierog et al., Phys. Rev. C 92034906 (2015).

[9] E. J. Ahn et.al., Phys. Rev. D 80094003 (2009).

[10] S. Ostapchenko, Phys. Rev. D 83014018 (2011).

[11] F. Riehn et.al., PoS ICRC 2017, 301 (2018).

[12] CMS Collaboration, arXiv:1903.12179 [hep-ex].

[13] M. Bähr et al., Eur. Phys. J. C 58639 (2008).

[14] J. Bellm et al., Eur. Phys. J. C 76196 (2016).

[15] S. Gieseke et al., Eur. Phys. J. C 77156 (2017).

[16] CMS Collaboration, JHEP 07032 (2018).

[17] CMS Collaboration, CMS-PAS-SMP-18-012 (2019), CDS Record 2668320.

[18] CMS Collaboration, JHEP 02032 (2018).

[19] G. Cowan et.al., Eur. Phys. J. C 711554 (2011).

[20] Read, Alexander L., J. Phys. G 282693 (2002).

[21] ATLAS and CMS Collaborations, CMS-NOTE-2011-005. ATL-PHYS-PUB-2011-11 (2011).

[22] J. R. Gaunt et al., Eur. Phys. J. C 6953 (2010).

[23] CMS Collaboration, JHEP 1403032 (2014). 